\title{
Virtual Geographic Environment Data Organization Model for Low- Altitude Collision Avoidance
}

\author{
Li Feng*, Xiao-hui Chen, Bin-chuan Jiang, and Li-heng Tan \\ Institute of Surveying and Mapping in Zhengzhou, Zhengzhou China \\ *Corresponding author: Feng Li, jueyun1020@126.com
}

\begin{abstract}
The geographical environment with low-altitude is complex. Ground mountain, tall buildings, communications tower, bridge across the river, the tall vegetation has a significant effect on low altitude fly. The technology of virtual navigation and collision warning system which based on satellite navigation and virtual geographic environment is an important technical guarantee for safety fly in low-altitude space. This paper analysed the characteristic of low-flying environment and its impact on safe flight. And designed a global virtual geographic environment data organization model based on global discrete three dimensional grid model combining with object-oriented data organization model. The way of subdivision, coding and object organization has been discussed, then designed the structure of the object in the model in low attitude environment. At last an algorithm for low-flying anti-collision warning was designed to prove use of this model.
\end{abstract}

Keywords: virtual geographic environments; collision detection; spatial information grid; low altitude collision avoidance

\section{Introduction}

Low altitude space is the airspace where altitude below 3000 meters, which does not affect air transport routes ${ }^{[1]}$. With the rapid development of our national economy, china's low-altitude airspace has gradually and orderly opening. Aircrafts in low-altitude will become more and more. According to conservative estimates, the number of general aviation aircraft in china will reach 18000 by 2020, which will drive the rapid development of related industries, thus will bring huge economic benefits, as well as challenge for the flight of the low altitude security ${ }^{[2]}$.The technology of visual navigation and collision avoidance warning which based on satellite navigation and virtual geographic environment isimportant technical support safety fly in low-altitude space. Low collision warning system need to real-time warn potential obstacles and provide a flight guidance, require real-time computing aircraft and ground buildings, communications tower, flying obstacles such as power lines, and the air zone, a no-fly zone and other special airspace spatial relations. The visual navigation of vehicles compares virtual scenes generated by computers to real scenes to help pilots' perception of flight environment, which requires to improve the fidelity of virtual geographical environment ${ }^{[3]}$. The traditional spatial data models of virtual geographic environment were designed for geology, mining, landscape areas, low altitude flight environment characteristics and low collision warning requirements were not considered. It is difficult to adapt to the characteristics of low altitude airspace without fixed boundary, a wide range, dynamic change. It is urgently to build low-level conflict avoidance oriented global multi resolution virtual geographical environment data organization model.

At present domestic related fields for low altitude collision avoidance applications have launched some research. Tan Xiao, Zhu Qing who come from Wuhan University have proposed complex space environment dynamic three-dimensional representation model of visual navigation of aircraft ${ }^{[4]}$. By the Tsinghua University, Wuhan University, Beihang University and other units jointly the national 973 project "under the complex conditions of aircraft approach visual navigation based theory", have researched the multidimensional dynamic complex space environment unified representation Led by Beihang University, part of the national 973 project "complex low flying self-hedging theory and 
method study", launched the research of low altitude complex environmental factors multi granularity relation representation and cognitive model ${ }^{[5]}$. But there is not any a mature model of data organization in the low altitude environment space at present. Aiming at the characteristics of low altitude environment and the needs of anti-collision alarm. This paper has designed a model of a low altitude data organization in global virtual geographical environment, through the experiment to verify the feasibility of the model.

\section{LOW ALTITUDE ENVIRONMENT SPACE DATA ORGANIZATION MODEL}

\subsection{Organization model design}

Low altitude aircraft usually do not follow specific routes are free to fly, resulting in low altitude airspace scope is broad and has no fixed boundaries. If the organization of environmental data in the map projections space rectangular coordinate system, will bring the projection deformation and map merging area crack problems, is not conducive to the organization and management of a wide range of low altitude data, therefore, need to directly in the spherical organization low altitude environment data.

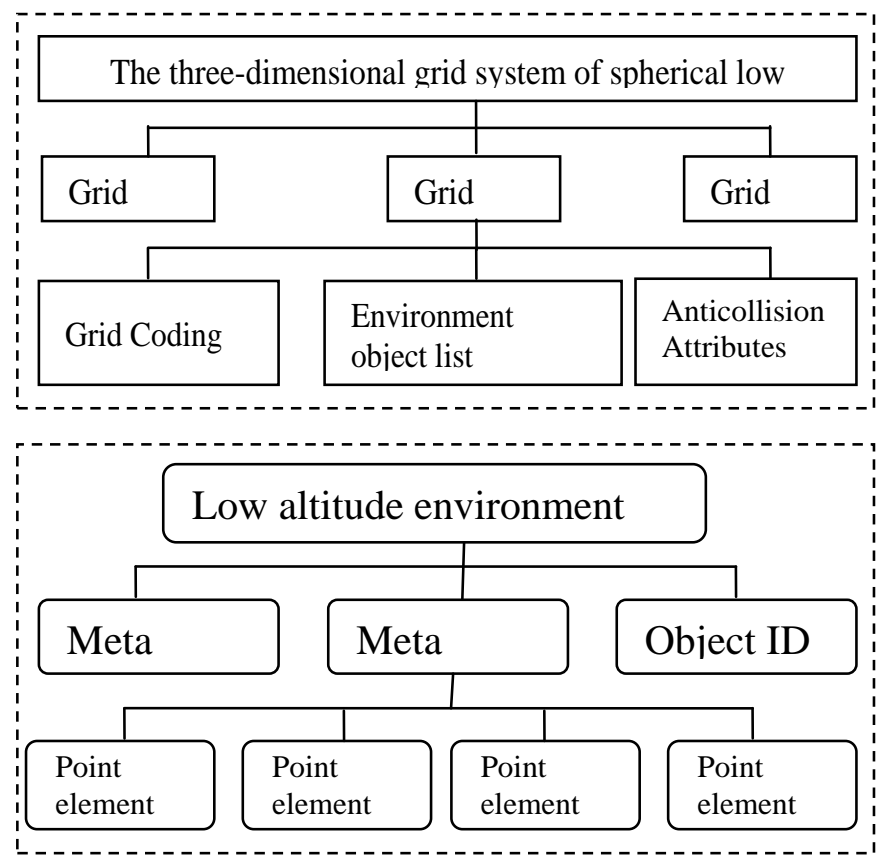

Fig.1 Schematic diagram of low altitude environmental data organization model

Low altitude environment data distributed in three-dimensional space, low altitude aircraft have a certain altitude, aircraft and environmental element spatial relation judgment requires use a threedimensional space data model, collision detection and path planning in three-dimensional space, require the precise collision model, spatial data to calculate will exponential growth. In order to improve the real-time collision avoidance algorithm, space data level of detail model is needed.

Environmental data of low altitude flight safety threat can be abstracted as independent of the environment entity, the entity objects with spatial characteristics, unique time characteristics and attributes. For low flying threats and threat degree and data updating mode are different, the object oriented technology to low altitude environment object classification processing, achieve anti-collision calculation, the respective data updating algorithm.

Based on the above analysis, low-level data organization model design of a spherical low altitude three-dimensional grid combined with object-oriented, unified management of low altitude multisource, multi temporal data, as shown in Fig. 1. 
Three dimensional grid is based on a spherical earth ellipsoid, the low altitude space is divided into a series of grid unit, a unique code is given to each grid unit, and gives the grid cell properties. The model of low altitude object oriented data model according to the spatial characteristics of a low altitude environment abstract to entity, as well as the collision warning algorithm and interface of update, judgment of the relationships between design of low-altitude environment object data.

\subsection{Spherical low altitude three-dimensional grid model}

A spherical low altitude three-dimensional grid model is to construct three dimensional grid system with hierarchical structure. Through the three-dimensional grid elements to describe the changes of spatial objects in low altitude environment. The nature of the spherical surface grid, and then a series of three-dimensional grid unit from earth radial division. At present, more scholars to break the plane model, storage, management, according to the real earth spatial information expression, many scholars have studied the spherical grid partition model, including grid system, classification based on latitude and longitude lines based on grid system, regular polyhedron partition based on voronoi grid system and hybrid grid system ${ }^{[6]-[7]}$. Spherical degenerate quad tree grid model is a latitude and longitude lines mesh, and grid cell geometry is simple. In addition to the top triangle, the rest are nearly rectangular grid unit, facilitating the adoption of longitude and latitude coordinates. Grid geometry parameters inthe subdivision level increases and the convergence, stability of deformation. To keep direction, radial symmetry and phase and in triangulation process, is conducive to the establishment of grid cell hierarchical index and efficiency of neighborhood search algorithm.

Extending to low space on the spherical based on quad tree degradation in the spherical surface grids, the hierarchical structure is divided into two parts to establish the radial in the radial direction, forming a "three-dimensional grid unit prismatic". Spherical degenerate quad tree grid model includes two kinds of spherical grid unit, the top triangle and quadrilateral. Accordingly, low-altitude threedimensional grid model also includes three-dimensional grid units like three prisms and four prisms. The large area located in a low latitude, so most of the grid unit is four prisms. To position grid unit accurately, we need the grid code for each unique three-dimensional grid unit, coding method of the grid cell is extended four fork tree trellis coded method of spherical surface degradation, the radial and spherical surface grid coding to form the 3D grid coding. Spherical low-altitude three-dimensional grid only grid of allarea of low space on the earth description. Through three-dimensional grid unit's setting covering the entire surface of low-altitude area, index ID established all kinds of information through the three-dimensional grid unit code.

A spherical low altitude three-dimensional grid system is designed to organize low altitude environment data, three tuple multi-source data structures can be effectively organized anti-collision property low altitude environment object and grid unit. The three tuple of multi-source data structure refers to the "three-dimensional grid coding -altitude change object -grid anti-collision properties" relational structure. Through the grid to the low-altitude environment object registration data object belongs to the specified grid units to realize the integration of multi-source data, through the integration of data object properties obtained in the three-dimensional grid unit grid stereo grid collision attributes. On the low-altitude environment object storage, low three-dimensional grid cell coding and low three-dimensional grid cell all kinds of attribute information "key value - record" structure, namely low three-dimensional grid cell coding SCode as key value, the corresponding records to fall into the low three-dimensional grid cell internal various attributes of the information collection of Ai, namely SCode\{ A1, $\cdots, A n\}$. Low altitude environment for different object, establish the corresponding relationship between the three dimensional grid unit set encoding covered in low altitude environment object identifier and low environmental objects, this is similar to "key record" structure, but the key became low altitude environment object identifier for the DataObjectID, 
and the record set low altitude three-dimensional grid unit code SCodei, DataObjectID \{SCode1,... , SCoden\}.

\subsection{Object-oriented data organization model of space environment design}

In the low altitude environment, low environmental factors affect flight safety can be abstracted as a spatial object, spatial objects according to classification standard to establish object identity only, and the spherical low altitude three-dimensional grid model association. The object oriented method in low altitude environment entity, for the real-time renewal movement object properties, convenient data model of multi resolution implementation.

First of all, the smallest unit will form a low environmental entity known as a low environmental meta Object (Mete Object, MO), meta Object is the basic unit of the rules of geometric satisfy geometric partitioning, cannot be subdivided with objective existence, is the minimum space unit has a realistic significance. Meta object consists of a set of limited set of discrete state to describe, each meta object is a quad (ID, GA, EA, MF).The ID is the unique identifier meta object, and in the low-level threedimensional grid model of spherical low-altitude environment object identifier DataObjectIDcorresponding;GA is a low environment entity space collection of properties (GeoAttribute), $\mathrm{GA}=\left\{\mathrm{GA}_{\mathrm{i}}, \mathrm{i}=1,2,3, \ldots, \mathrm{n}\right\}$;EA collection of non-spatial attributes is a low environmental Entity (Entity Attribute), $\mathrm{EA}=\left\{\mathrm{EA}_{\mathrm{i}}, \mathrm{i}=1,2,3, \ldots, \mathrm{n}\right\} ; \mathrm{MF}$ is a low environmental entity a collection of all the Methods (the Methods Function), for the collision model building provides a common interface. Secondly, will constitute a geometric feature abstracted as point, low altitude environment element object, class three, and known as the geometric elements (Geometry, Element, GE). Some elements $\mathrm{P}=\{\mathrm{PID}, \mathrm{X}, \mathrm{Y}, \mathrm{Z})$; line element $\mathrm{L}=\left\{\mathrm{LID}, \mathrm{PID}_{1}, \mathrm{PID}_{2}, \mathrm{PID}_{3}, \ldots, \mathrm{PID}_{\mathrm{n}}, \ldots\right.$ ); surface elements $\mathrm{A}=\{\mathrm{AID}$, $\mathrm{LID}_{1}, \mathrm{LID}_{2}, \mathrm{LID}_{3}, \ldots, \mathrm{LID}_{\mathrm{n}}, \ldots$ ). Low environmental meta object in space formed by the geometrical element such as dot, line, face, namely the $\mathrm{MQ}=\{\{\mathrm{P}\},\{\mathrm{I}\},\{\mathrm{A}\}\}$, A low environmental space attribute of the meta object can be described by A series of geometric elements directly, also can be an external import model description, such as ordinary overhead pipeline is by the description of point, line and plane, while the tall buildings of landmark by the import external fine $3 \mathrm{~d}$ model to describe. Finally, the low level environmental entity information for storage, management and application of the subject is called space objects (Spatial Object, SO), space Object consists of a series of meta Object, can be expressed as: $\mathrm{SO}=\left\{\mathrm{MO}_{1}, \mathrm{MO}_{2}, \mathrm{MO}_{3}, \ldots, \mathrm{MOn}, \ldots\right\}$.

\section{LOW ALTITUDE ANTI-COLLISION ALGORITHM}

The key of low altitude aircraft collision warning system is real-time alarm algorithm to design efficient. Aircraft from the sensor or data link realtime receiving position, velocity, attitude and dynamic changes in the environment information, the information is updated by the interface method of internal attributes of spatial objects. Low level dynamic target acquisition through the data chain, dynamic registration to the spherical low altitude three-dimensional grid, aircraft position, attitude, velocity information obtained by sensors. According to the above information can be calculated based on the structure of spherical warning distance, low altitude three-dimensional grid model implementation of initial collision filtering fast, then according to the method of exact collision low altitude environment object storage of three dimensional grid model.

The algorithm based on low level environmental database and real-time navigation and positioning information, real-time early warning aircraft flying low potential threats in the process, the key step in the algorithm implementation is as follows:

(1)early warning range in low altitude and numerous threats object, but only warning within the scope of the object need to participate in the calculation. Warning range is according to the current speed, the position of the aircraft, and flight direction to determine, and flight speed were positively ratio, and is inversely proportional flying height. 
(2)based on low level three-dimensional grid data collision roughly filtering.Low three-dimensional grid with anti-collision attributes, judge into attributes of collision avoidance warning range cell, can quickly extract the potential threat of a grid cell.

(3)based on a low environment object data accurate collision detection.In view of the grid cell potential threats, according to the space object ID to extract low environment object data, and collisions with geometrical intersection way precise calculation.

(4)collision warning. If according to the craft of the current state of collision risk, with voice, image, light alarm, and avoid dangerous advice is given.

\section{EXPERIMENT AND CONCLUSION}

To verify the validity of the model, the "aviation service station monitoring alarm system" has realized the low collision warning.System in Shanghai area for experimental data, the establishment of the lowlevel three-dimensional grid model, the tall buildings, communications tower, aerial pipeline, the bridge across the river as a space object entity management, and divided into three-dimensional grid, will the air zone, and a no-fly zone data entry in the system. Through real-time server receives ads-b back to the area of general aviation aircraft information, update the database in real time.System load flight path in advance, the aircraft flying current state with HUD displayed in the navigation on tablets, the alarm way with the method of voice and image geometry, did an experiment system on the helicopter application, application effect is shown in Fig.2.

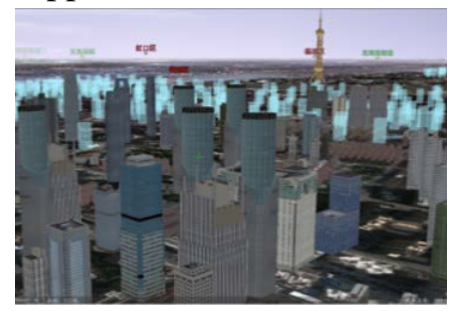

a urban Building management

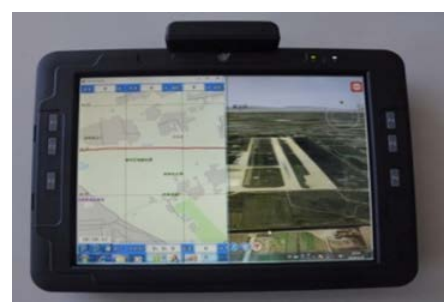

c Tablet PC navigator

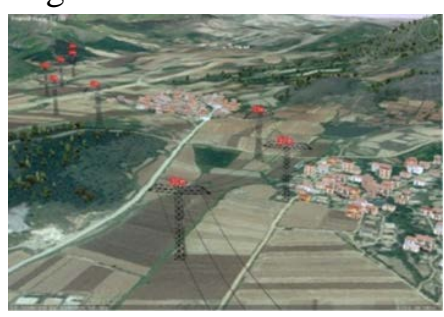

b High voltage wire management

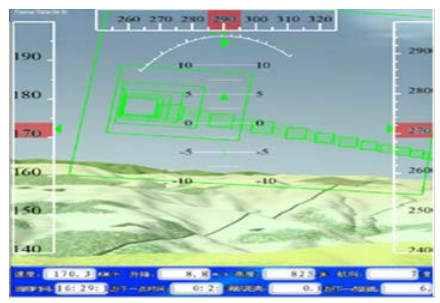

$d$ The flight path displaying

Fig.2 Aviation Service Station experimental effect monitoring alarm system

\section{Acknowledgements}

This thesis is Supported by the National Natural Science Foundation of China (41301428) and State Key Laborary of Geo-information Engineering,NO SKLGIE2015-Z-4-1.

\section{References}

1. W. Wang,About the problem of China's low altitude airspace management thinking of [J]. air traffic management, 20012,1 (2):182-186. 
2. B. Sykora,Rotorcraft vitual situation awarenesssolving the polotage for landing in degraded vistual environments[C], The American Helicopter society 65th Annual Forum. Grapvine, Texas:BAE Systems, 2009

3. X.Tan, Q.Zhu, J.J.Zhao, W.Xu, Y.Zhang, Z.Du. The complex space environment dynamic 3D representation model [J]. Journal of Surveying and mapping for vehicle visual navigation, 2012,41 (2): 177-183.

4. http://media.au.tsinghua.edu.cn/973website/default.htm

5. Ben. Aperture for global hexagonal grid system index method 4 [J]. Journal of Surveying and mapping, 2011,40 (6): 785-789.

6. $\quad \mathrm{L}$. $W u$,Earth grid and its application model system of spatial geography and geo information science[J], 2012,28 (1): 7-13.

7. X.Zhao, Global diamond block discrete grid hierarchical modeling based on [J]. Journal of China University of Mining and Technology, 2007,36 (3): 398-401. 